PR-23

\title{
SYNTHESIS OF CARBOXAMIDE AND SULFONYL CARBOXAMIDE LINKED AZOLES UNDER GREEN CONDITIONS
}

\author{
G. Lakshmi Teja, ${ }^{1}$ T. Rekha, ${ }^{1}$ G. Sravya ${ }^{2}$ and V. Padmavathi ${ }^{1}$ \\ ${ }^{1}$ Department of Chemistry, Sri Venkateswara University, Tirupati, Andhra Pradesh, India. \\ ${ }^{2}$ Ural Federal University, Chemical Engineering institute, Yekaterinburg, \\ 620002, Russian Federation. \\ Corresponding author, E-mail: vkpuram2001@yahoo.com
}

\begin{abstract}
Amide and sulfonamide functionalities represent privileged structural motifs and play prominent role in the composition of polymers, proteins, natural products and appear in more than $25 \%$ of known drugs. Various approaches were explored for efficient construction of this significant skeleton. The most common synthetic routes relay on the reactions of activated carboxylic acids and their derivatives with amines, Beckmann rearrangement, StaudingerVilarrasa reaction, amidation of aryl halides etc., However, these methods have innate drawbacks, for instance, a large amount of by products are generated leading to lower yields. As such there is a quest for efficient and economically viable methods to construct amide bond. Besides azoles are prominent players in pharmaceutical arena. In continuation of our interest on green-reaction conditions for organic transformations, a simple and efficient protocol for the synthesis of carboxamide and sulfonyl carboxamide linked azoles was developed using PEG-400 in the presence of an oxidant $\mathrm{CCl}_{3} \mathrm{CN} / \mathrm{H}_{2} \mathrm{O}_{2}$ as eco-friendly and recyclable medium. The results pertaining to these reactions will be presented.
\end{abstract}

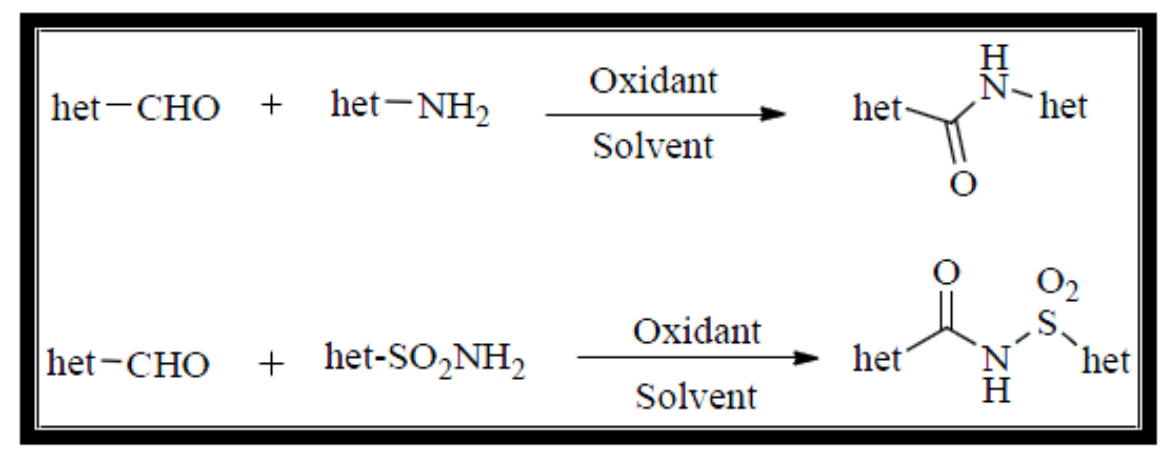

